This item was submitted to Loughborough's Research Repository by the author.

Items in Figshare are protected by copyright, with all rights reserved, unless otherwise indicated.

\title{
Helium bubbles in Fe: equilibrium configurations and modification by radiation
}

PLEASE CITE THE PUBLISHED VERSION

http://dx.doi.org/10.1557/opl.2013.198

\section{PUBLISHER}

(C) Materials Research Society. Published by Cambridge University Press.

\section{VERSION}

AM (Accepted Manuscript)

\section{PUBLISHER STATEMENT}

This work is made available according to the conditions of the Creative Commons Attribution-NonCommercialNoDerivatives 4.0 International (CC BY-NC-ND 4.0) licence. Full details of this licence are available at: https://creativecommons.org/licenses/by-nc-nd/4.0/

\section{LICENCE}

CC BY-NC-ND 4.0

\section{REPOSITORY RECORD}

Gai, Xiao, Roger Smith, and Steven D. Kenny. 2015. "Helium Bubbles in Fe: Equilibrium Configurations and Modification by Radiation”. figshare. https://hdl.handle.net/2134/19684. 


\title{
Helium Bubbles in Fe: Equilibrium Configurations and Modification by Radiation
}

Xiao Gai, Roger Smith and Steven Kenny

Mathematical Sciences Department, Loughborough University, Leicestershire, LE11 3TU, UK

\begin{abstract}
We have examined the properties of helium bubbles in Fe using two different Fe-He potentials. The atomic configurations and formation energies of different He-vacancy complexes are determined and their stability in the region of nearby collision cascades is investigated. The results show that the optimal $\mathrm{He}$ to $\mathrm{Fe}$ vacancy ratio increases from about 1:1 for approximately 5 vacancies up to about 4:1 for 36 vacancies. Collision cascades initiated near the complex show that $\mathrm{Fe}$ vacancies produced by the cascades can be added to it more easily than $\mathrm{Fe}$ interstitials. The energy barrier for an isolated He interstitial to diffuse was found to be $0.06 \mathrm{eV}$. Thus a possible mechanism for $\mathrm{He}$ bubble growth would be the addition of vacancies during a radiation event, induced by a nuclear reaction, followed by the subsequent accumulation of mobile $\mathrm{He}$ interstitials.
\end{abstract}

\section{INTRODUCTION}

Reduced-activation ferritic/martensitic steels are candidate materials for use in nuclear reactors. The presence of transmutation-created helium plays an important role in the microstructural evolution of these steels under neutron irradiation. Helium has a large effect on cascade damage: substitutional He recombines with self interstitial atoms (SIAs), leading to fewer surviving defects, while interstitial helium binds strongly to SIA clusters, stabilising and trapping them, leading to more surviving defects.

Small helium-vacancy $\left(\mathrm{He}_{\mathrm{n}} \mathrm{V}_{\mathrm{m}}\right)$ clusters may play an important role in the nucleation of $\mathrm{He}$ bubbles. However, the atomistic properties of $\mathrm{He}$ in metals are difficult to identify experimentally. Thus atomistic simulations such as molecular dynamics (MD) provide useful tools to study the formation and the stability of these clusters. Here, we present the results of a study on the formation of small helium-vacancy clusters in bcc iron and their interaction with nearby collision cascades, which will provide insight into the growth of the bubble.

\section{COMPUTATIONAL METHOD}

The formation energies of the helium-vacancy clusters $\mathrm{He}_{\mathrm{n}} \mathrm{V}_{\mathrm{m}}$ are evaluated using different empirical potentials. For the Fe-He potential, we use two different ones. The first is a three body Fe-He potential [1]. The other potential contains many body parts [2]. The first one is combined with the 1997 Ackland et al. potential [3] for Fe while the latter one uses the Ackland and 
Mendelev potential (AM-potential) [4]. Here, the formation energy is defined as the difference in total energy between a crystal containing a defect and a perfect crystal of the same number of Fe atoms with the corresponding number of helium atoms in a fcc structure.

$$
E_{f}=E_{b}+(n-m) E_{v a c}-N_{F e} E_{F e}-n E_{H e}^{s u b}
$$

where $E_{f}$ represents the formation energy. $E_{b}$ represents the energy of the lattice containing the bubble; $E_{v a c}$ is the formation energy of a single vacancy in bcc-iron, which is defined as the difference between the energy of a bcc-Fe lattice of (N-1) atoms containing one vacancy and the energy of a perfect Fe crystal with N-1 atoms.

$$
E_{v a c}=E(v, n-1)-(n-1) E_{F e}
$$

$N_{F e}$ is the number of Fe atoms in the lattice containing the bubble. $E_{F e}$ is the cohesive energy of Fe. $E_{F e}^{s u b}$ is the energy of a helium substitutional atom, defined in He as follows,

$$
E_{H e}^{s u b}=E_{s u b}^{r e f}-N_{F e}^{r e f} E_{F e} .
$$

This is the difference between the energy of a reference bcc Fe lattice containing a substitutional $\mathrm{He}$ and the number of $\mathrm{Fe}$ atoms in the reference lattice multiplied by the cohesive energy of Fe.

In the calculations for the formation energy, the box size is set to $30 a_{0} \times 30 a_{0} \times 30 a_{0}$, where $a_{0}$ is the lattice parameter. For all calculations periodic boundary conditions and constant volume are used. The clusters have been generated with the following procedure. We start by creating the pure bcc Fe lattice, then remove atoms to get an approximately spherical vacancy cluster. Next, generate close packed He of approximately the same size as the vacancy. Move He into the Fe lattice. The system is relaxed using a conjugate gradient algorithm. For each ratio several initial random configurations are tested and the one with the lowest formation energy is kept.

To simulate the cascade event, molecular dynamics is used. The system is first thermalised before a cascade event. We choose a value of $500 \mathrm{~K}$ for the system temperature, similar to reactor operating conditions. After that a $1 \mathrm{keV}$ cascade is initiated near the bubble. This is achieved by imparting $1 \mathrm{keV}$ energy to a primary knock-on atom (PKA). To ensure results are reliable, cascades must rigorously sample all unique directions within the crystal. With cubic systems, this calculation can be reduced somewhat due to high symmetry resulting in a resulting in an irreducible volume that is deemed representative of the whole structure but for He bubbles the symmetry is destroyed. Figure 1 illustrates the range of trajectories chosen for the calculation and the three positions chosen for the PKA. 


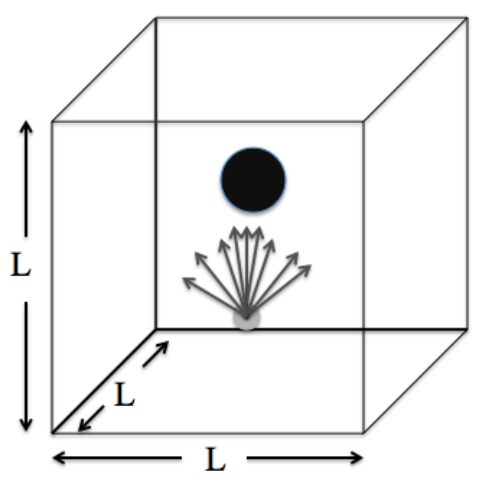

Fig. 1 The choice of directions for the collision cascades. The black ball represents the $\mathrm{He}$ bubble, whose centre is placed offset by about half the lattice constant from the centre of the box. The grey sphere represents the PKA, with arrows signifying the directions of the trajectories. The position of PKA here is $(0.3 \times \mathrm{L}, 0.5 \times \mathrm{L}, 0.5 \times \mathrm{L})$ but positions at $(0.3 \times \mathrm{L}, 0.4 \times \mathrm{L}, 0.5 \times \mathrm{L})$ and $(0.2 \times \mathrm{L}$ $0.5 \times \mathrm{L}, 0.5 \times \mathrm{L})$ are also chosen. $\mathrm{L}$ is the length of the box size, which is equal to $30 \mathrm{a}_{0}$.

In all 66 directions are selected. Furthermore, for ensuring reasonable statistics, different times of thermalisation are used. In addition to the cascade calculations we also determined the energy barrier for an isolated He atom to diffuse in a perfect bcc Fe lattice.

\section{RESULTS}

\section{Formation Energy}

Fig. 2 shows the formation energy calculated from the two different Fe-He potentials. Both empirical potentials reveal the same trends; that is to say there is an optimal He to vacancy ratio for each curve (calculated with a fixed number of vacancies). The formation energy decreases inserting more helium atoms into the vacancy unit until the cluster reaches an optimal size. The optimum ratio increases from around $1: 1$ for small bubbles up to $4: 1$ for large bubbles. When comparing the two potentials, it can be seen that the three body potential gives a formation energy at least $1.5 \mathrm{eV}$ higher than the many body potential except for the extremely small clusters (with 2 vacancies) and the optimal ratio for the many body potential is slightly higher than for the three body potential. The energy barrier for isolated He to diffuse was found to be very small at $0.06 \mathrm{eV}$ and therefore the diffusion is observable on MD time scales. 


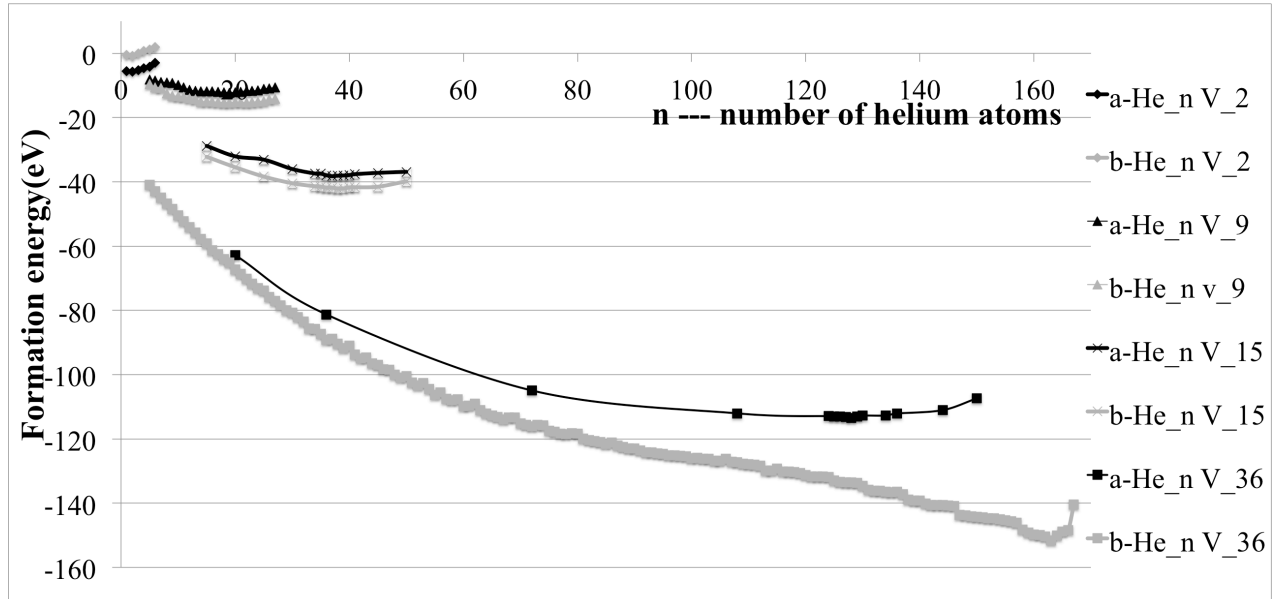

Figure 2. The formation energy as a function of the number of helium atoms for different sizes of $\mathrm{He}_{\mathrm{n}} \mathrm{V}_{\mathrm{m}}$ clusters. Here, a represents the three body potential for $\mathrm{Fe}-\mathrm{He}$ interactions while $\mathrm{b}$ is for the many body potential.

\section{Collision Cascades}

Both the two potentials demonstrate the conclusion that collision cascades can increase the size of the helium bubble. We can calculate the volume of the helium bubble as a function of time. Fig. 3 shows some typical examples.

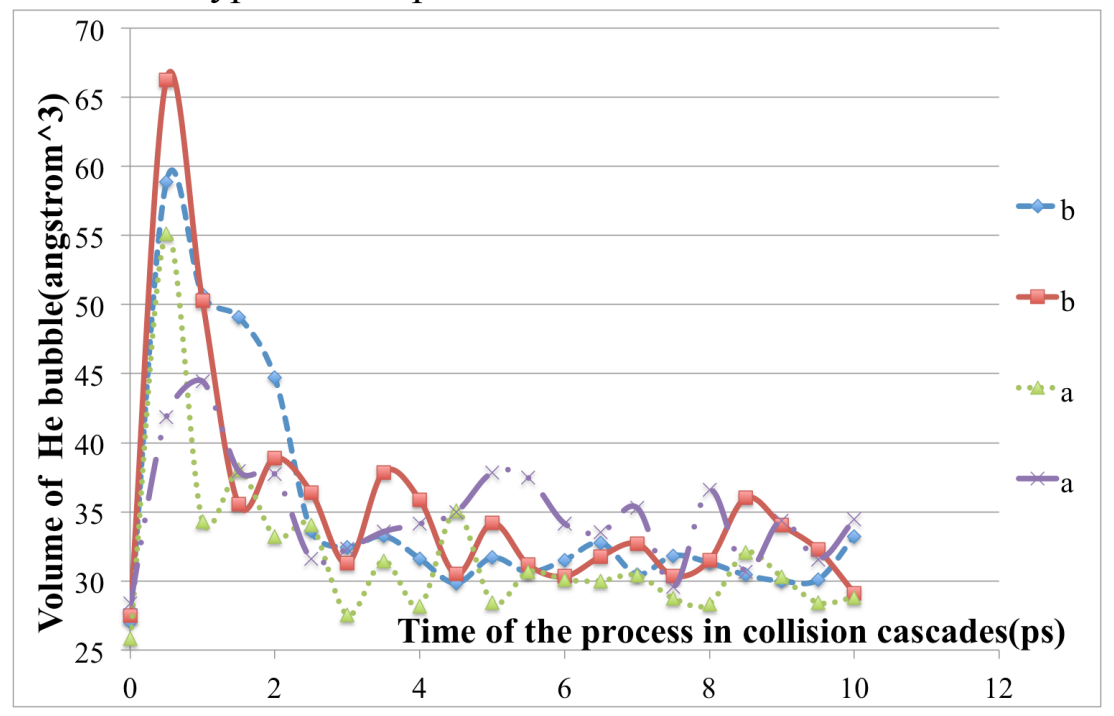

Figure 3. The curves show the volume of He bubble against the time. Here are just some examples from the samples.

Fig. 3 shows that during the collisional process, especially at the beginning, the cascade will cause the expansion of the helium bubble, then the bubble contracts as the time goes on and finally its volume becomes stable. Moreover, we find that the volume of the helium bubble is increased compared to the original one when the cascades pass through it while the volume does 
not change so much if the cascade is far away from the bubble.

In order to determine the reason for the change in bubble size we examine the increase/decrease in Fe vacancies in the bubble by statistical averaging over all the generated cascades. Fig. 4 and Fig. 5 show examples for three helium-vacancy clusters where the number of vacancies is kept constant.

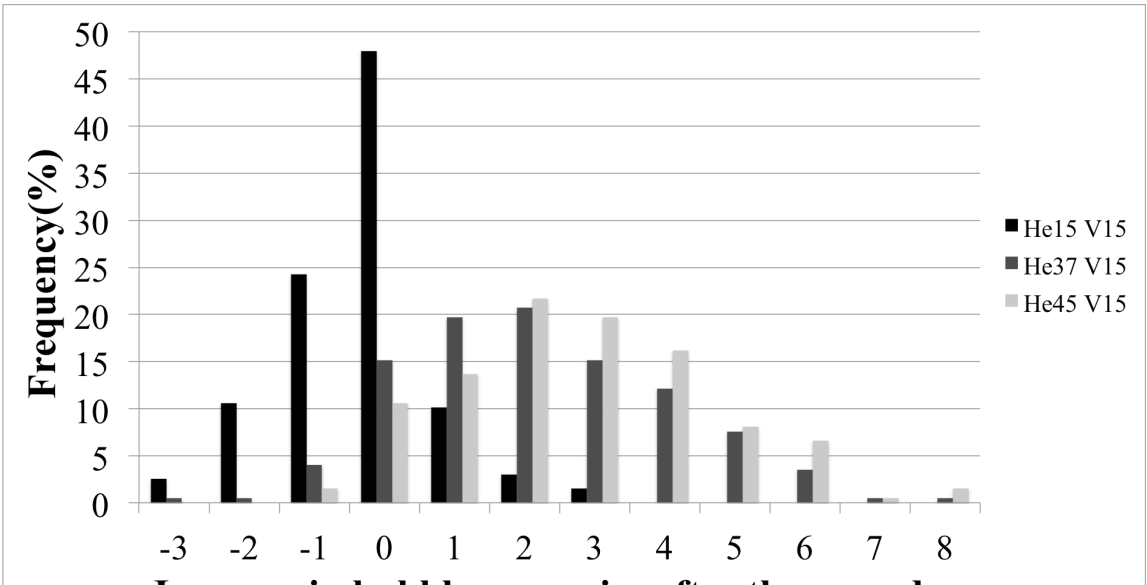

Increase in bubble vacancies after the cascade

Figure 4. Statistics for the differences between Fe interstitials and vacancies using the three body potential. The horizontal axis shows the number of He atoms -the number of vacancies in the He complex averaged over the $66 \times 3$ separate cascades. The vertical axis is the frequency of occurrence.

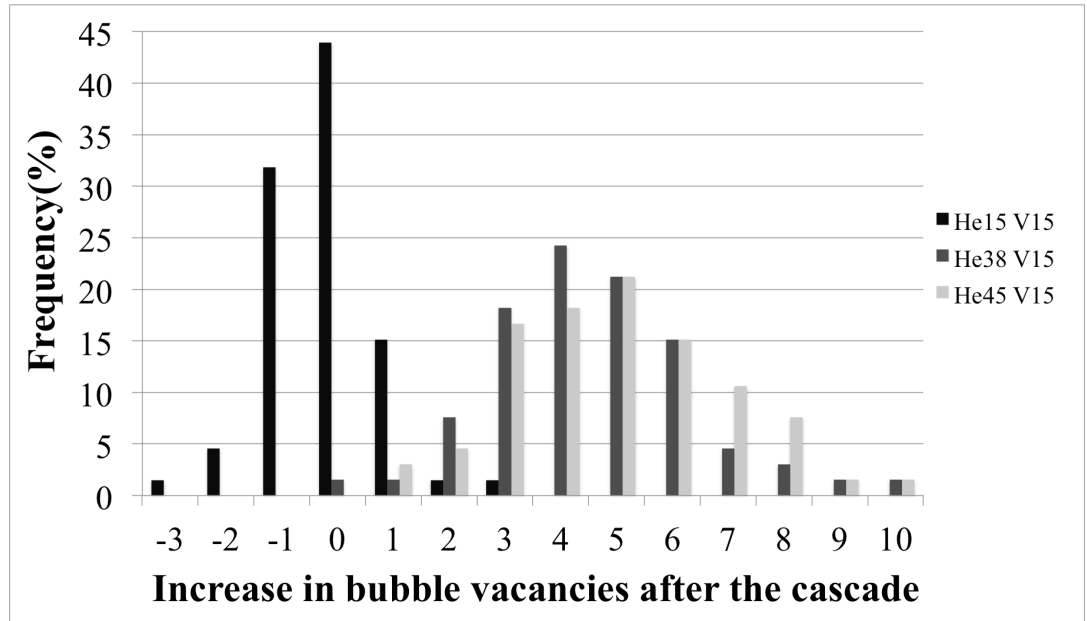

Figure 5. This is the same calculation as in figure 4 but for the many body potential.

As might be expected, at low helium-to-vacancy ratio, the emission of vacancies is clearly favoured. This changes at an optimal helium-to-vacancy ratio, where the cascade can cause the emission of Fe interstitials. When the helium density increases again, this phenomenon becomes more obvious. Both Fe-He potentials demonstrate this conclusion with the many body potential, showing the effect more strongly than the three body potential. Above the optimal ratio, helium 
dissociation and the emission of interstitials become more favoured. Figure 6 shows a typical example.
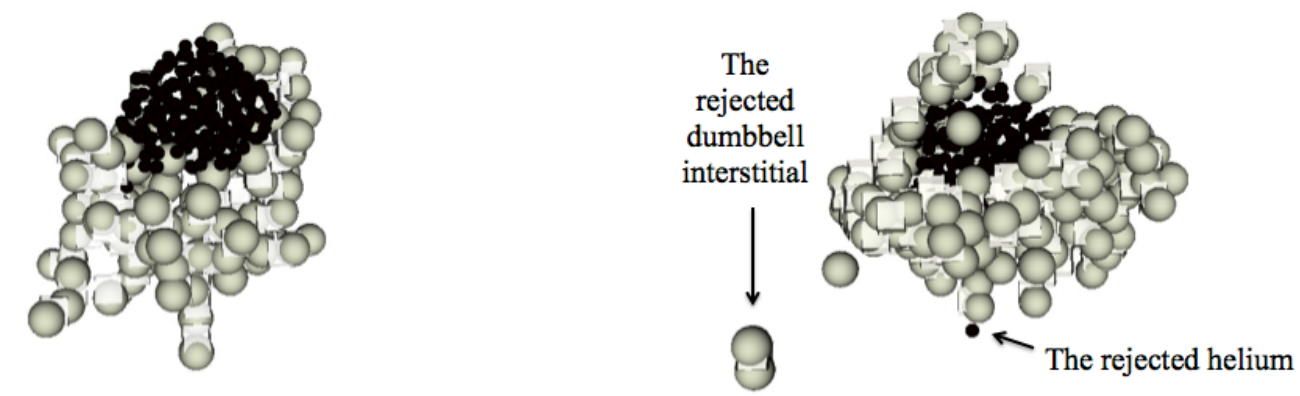

Figure 6. Two frames in the simulation of a collision cascade near a $\mathrm{He}_{200} \mathrm{~V}_{36}$ complex using the many body potential. The left one is after $100 \mathrm{fs}$ just as the cascade reaches the bubble while the right one is after $1500 \mathrm{fs}$ when the cascade has passed into the bubble.

\section{CONCLUSIONS}

The stability of helium vacancy clusters in bcc iron has been investigated using static atomistic simulation and molecular dynamics. Two potential functions are compared which show similar results for the formation energies of the He- vacancy clusters. As might be expected in the presence of collision cascades He-vacancy complexes with a sub optimal ratio of He atoms absorb Fe interstitials but for optimal and super optimal ratios the cascades cause the emission of Fe interstitials from the complexes more favourably. Furthermore, a very low migration energy $(0.06 \mathrm{eV})$ for diffusion of interstitial $\mathrm{He}$ in $\mathrm{Fe}$ is obtained, which shows the helium atoms can diffuse over MD time scales. A possible mechanism for the growth of He bubbles is therefore the creation of excess vacancies in the complexes by irradiation followed by the diffusion of isolated He interstitials.

\section{ACKNOWLEDGMENTS}

This work was carried out with the support by EPSRC's PROMINENT project.

\section{REFERENCES}

1. R. E. Stoller, S. I. Golubov, P. J. Kamenski, T. Seletskaia, Yu. N. Osetsky, Phil. Mag. 90(2010) 923-934

2. F. Gao, Huiqiu Deng, H. L. Heinisch, R. J. Kurtz, J. Nucl. Mater., 418(2011) 115-120

3. G. J. Ackland, D. J. Bacon, A. F. Calder and T. Harry, Phil. Mag. A 75(1997) p.713

4. G. J. Ackland, M. I. Mendelev, D. J. Srolovitz, S. Han, A. V. Barashev, J. Phys.: Condens. Mater, 16(2004) S2629 